\title{
A search for VHE counterparts of Galactic Fermi bright sources and MeV to TeV spectral characterization
}

\author{
P. H. T. Tam ${ }^{1, \star}$, S. J. Wagner ${ }^{1}$, O. Tibolla ${ }^{2}$, and R. C. G. Chaves ${ }^{2}$ \\ ${ }^{1}$ Landessternwarte, Universität Heidelberg, Königstuhl, 69117 Heidelberg, Germany \\ e-mail: phtam@lsw.uni-heidelberg.de \\ 2 Max-Planck-Institut für Kernphysik, PO Box 103980, 69029 Heidelberg, Germany
}

Received 23 November 2009 / Accepted 21 March 2010

\begin{abstract}
Very high-energy (VHE; $E>100 \mathrm{GeV}$ ) $\gamma$-rays have been detected in a wide range of astronomical objects, such as pulsar wind nebulae (PWNe), supernova remnants (SNRs), giant molecular clouds, $\gamma$-ray binaries, the Galactic center, active galactic nuclei (AGN), radio galaxies, starburst galaxies, and possibly star-forming regions. At lower energies, observations using the Large Area Telescope (LAT) onboard Fermi provide a rich set of data that can be used to study the behavior of cosmic accelerators in the MeV to TeV energy bands. In particular, the improved angular resolution of current telescopes in both bands compared to previous instruments significantly reduces source confusion and facilitates identification of associated counterparts at lower energies. In this paper, a comprehensive search for VHE $\gamma$-ray sources that are spatially coincident with Galactic Fermi/LAT bright sources is performed, and the available $\mathrm{MeV}$ to $\mathrm{TeV}$ spectra of coincident sources compared. It is found that bright LAT GeV sources are correlated with $\mathrm{TeV}$ sources, in contrast to previous studies using EGRET data. Moreover, a single spectral component seems unable to describe the $\mathrm{MeV}$ to TeV spectra of many coincident $\mathrm{GeV} / \mathrm{TeV}$ sources. It has been suggested that $\gamma$-ray pulsars may be accompanied by VHE $\gamma$-ray emitting nebulae, a hypothesis that can be tested with VHE observations of these pulsars.
\end{abstract}

Key words. Gamma rays: general - Galaxy: general - pulsars: general - ISM: supernova remnants - X-rays: binaries

\section{Introduction}

Our understanding of the very high-energy (VHE; $E>100 \mathrm{GeV}$ ) sky has greatly improved during the last few years, thanks to the high sensitivity of current imaging atmospheric Cherenkov telescopes (IACTs), e.g., HESS, MAGIC, and VERITAS. They typically cover the energy range of $\sim 100 \mathrm{GeV}$ up to several tens of $\mathrm{TeV}$, and provide an angular resolution of $\sim 6^{\prime}$. This allows spectral and morphological studies of the various types of VHE sources: pulsar wind nebulae (PWNe), supernova remnants (SNRs), giant molecular clouds, $\gamma$-ray binaries, the Galactic center, active galactic nuclei (AGN), radio galaxies, starburst galaxies, and possibly star-forming regions. See Aharonian et al. (2008) for a review of the field in 2008, with more recent updates given by the HESS (Chaves et al. 2009a), MAGIC (Zanin et al. 2009), and VERITAS collaborations (Ong et al. 2009; Weinstein et al. 2009). However, many of the sources have not yet been identified at other wavelengths; e.g., nearly a third of the Galactic HESS sources have no firm identification, and in many cases, there are multiple plausible counterparts while in others, no viable counterparts have been identified.

Gamma-ray observations of Galactic sources can help us solve a number of important astrophysical questions, including (1) the physics of pulsars, PWN, and SNR; and (2) the origin of cosmic rays. Our Galaxy contains several cosmic accelerators, where particles are accelerated to highly-relativistic energies (up to at least $10^{14} \mathrm{eV}$ ). The origin of cosmic rays is still not well

^ Now at Institute of Astronomy and Department of Physics, National Tsing Hua University, Hsinchu 30013, Taiwan. known, largely because of the lack of directional information of these particles. These very energetic particles can be traced within our Galaxy by a combination of nonthermal X-ray emission and $\gamma$-ray emission via leptonic (such as inverse Compton scattering of electrons, Bremsstrahlung and synchrotron radiation) or hadronic (via the decay of charged and neutral pions, due to interactions of energetic hadrons) processes. Therefore, observations of $\gamma$-rays at energies $\gtrsim 100 \mathrm{MeV}$ can probe the sources of particle acceleration.

The Large Area Telescope (LAT), onboard the Fermi Gamma-ray Space Telescope, provides the best information of the nonthermal sky in the energy range from $20 \mathrm{MeV}$ to $300 \mathrm{GeV}$. The point-source sensitivity of LAT is $\sim 10^{-8} \mathrm{ph} \mathrm{cm}^{-2} \mathrm{~s}^{-1}$ above $100 \mathrm{MeV}$ in one year of surveymode observations (Atwood et al. 2009), which is an order of magnitude better than that of its predecessor, the Energetic Gamma Ray Experiment Telescope (EGRET). Its angular resolution is $\lesssim 0^{\circ}$. 6 above $1 \mathrm{GeV}$, which is particularly important for identifying $\gamma$-ray sources with multi-wavelength counterparts and revealing their nature (Atwood et al. 2009). As an important step towards the first source catalog, the LAT collaboration has published a bright source list (BSL) that includes 205 sources, designated with the prefix OFGL, using data taken during the first three months of observations (Abdo et al. 2009a). Among them, 121 sources are identified with AGN and one with the Large Magellanic Cloud. Most of the remaining 83 sources are believed to have originated in our own Galaxy. It is natural to investigate which of them also have been detected at energies $\gtrsim 100 \mathrm{GeV}$. 
The search for VHE counterparts of LAT sources is important for the following reasons:

1. it aids the identification of the true nature of the LAT sources through their VHE counterparts;

2. for pulsars, it helps us to identify their VHE-emitting nebulae;

3. it may provide us with broad-band $\gamma$-ray spectra, thereby better constraining the emission mechanisms (e.g. distinguish between hadronic and leptonic scenarios).

Funk et al. (2008) compare $\gamma$-ray sources in the third EGRET (3EG) catalog (Hartman et al. 1999) and the 22 HESS sources known at the time within the region of $l=-30^{\circ}$ to $30^{\circ}, b=-3^{\circ}$ to $3^{\circ}$ (Aharonian et al. 2006f). They do not find any spatial correlation between the two populations. Though some coincidence cases are found, the authors conclude that these few cases can be explained by coincidence. However, thanks to the capabilities of EGRET, this study suffers from the following limitations. (1) The sensitivity of EGRET is lower than that of LAT. The lack of photon statistics leads to poorly constrained spectral indices and the spectra terminate $\lesssim 10 \mathrm{GeV}$ at the upper end for a typical source. (2) EGRET sources are only localized at degree scales, which is much greater than the angular resolution of IACTs. The second point is the instrumental reason, which explains the weak correlation of EGRET and HESS sources (Funk et al. 2008). These shortcomings are now largely overcome by the enhanced performance of LAT over EGRET. In addition to the above caveats, they do not consider the extension of the VHE $\gamma$-ray sources in their analysis. As such, the full potential of this search has not been realized for very extended sources like the SNR RX J1713.7-3946, as pointed out by Tibolla et al. (2009a). After the launch of LAT, one largely benefits from the increased LAT angular resolution over previous studies. As noted in Atwood et al. (2009), EGRET could not distinguish the GeV emission of RX J1713.7-3946 from 3EG J1714-3857, while the capabilities of LAT allow study of individual sources in this region, which contains three VHE $\gamma$-ray sources (see Fig. 1 in Aharonian et al. 2008b).

The water Cherenkov detector MILAGRO covers the energies above $\sim 1 \mathrm{TeV}$, and its angular resolution can reach $<1^{\circ}$. Using MILAGRO, a search for $\gamma$-rays from the Galactic LAT BSL was performed by Abdo et al. (2009g). They find that 14 sources (of the selected 34 ) show evidence of multi-TeV $\gamma$-ray emission at a significance of $\geq 3 \sigma$, although most of the source candidates cannot be established as firm detection on an individual basis (Abdo et al. 2009g).

In this paper, a search for VHE counterparts of all the presumed Galactic sources in Abdo et al. (2009a) and Abdo et al. (2010) is performed, with spatial coincidence as the primary criterium for association. The extensions of the VHE $\gamma$-ray sources are taken into account, and the search is not limited to the HESS Galactic plane survey region. The broad-band $\mathrm{MeV}$ to TeV spectra of coincident sources are then presented.

\section{Search of spatial coincidence}

\subsection{The Fermi and VHE catalogs}

Abdo et al. (2009a) present 205 point-like sources that were detected at or above the $10-\sigma$ level in the $0.2-100 \mathrm{GeV}$ band, based on three months of observations (August 4, 2008-October 30, 2008). The list is not flux-limited, and so is not uniform. The following information is given for each source: its position, positional uncertainty (95\% confidence level, C.L.), significance, flux in two energy bands (100 MeV-1 GeV and 1-100 GeV), and any evidence of variability over the above-mentioned period. In addition, Abdo et al. (2009a) assign the source class for each source, as well as $\gamma$-ray and lower energy association (if any). Those sources that are classified as extragalactic (all AGN and the Large Magellanic Cloud) are not considered in this work.

The remaining source list contains 83 sources, comprising 15 radio/X-ray pulsars, 15 pulsars newly discovered by the LAT, two high-mass X-ray binaries (HMXBs), one globular cluster (47 Tucanae), 13 SNR/PWN candidates ${ }^{1}$, and 37 sources without obvious counterparts at lower energies (among them the Galactic center; Abdo et al. 2009a). Abdo et al. (2010) presents the first LAT pulsar catalog. Those 16 pulsars that are not present in the above BSL are also included in this study. Therefore, most of the LAT bright sources considered in this work should be Galactic in origin.

There are more than 50 VHE $\gamma$-ray sources as of Fall 2009 (Aharonian et al. 2008e; Chaves et al. 2009a; Zanin et al. 2009; Ong et al. 2009; Weinstein et al. 2009). Galactic sources summarized in the above publications are used in the search for coincidence in this work. Therefore, our comparison is only based on published sources. The VHE $\gamma$-ray source positions and source extensions in this work have been taken from the corresponding publications shown in Tables 1,2, and 3. At higher energies, the MILAGRO collaboration reported evidence of multi$\mathrm{TeV} \gamma$-ray emission from several LAT source positions (Abdo et al. 2009g). Only those source candidates with a significance greater than $5 \sigma$ are regarded as $\mathrm{TeV}$ sources here and are included in this study ${ }^{2}$. With several tens of known sources in both the $\mathrm{GeV}$ and $\mathrm{TeV}$ bands, a systematic cross-correlation study can be conducted.

\subsection{Level of spatial coincidence}

To quantify the level of spatial coincidence, the following scheme is employed. Let $d$ be the distance between a centroid position best fit by LAT and the best-fit centroid of a nearby VHE $\gamma$-ray source. The radius of the $95 \%$ confidence region for the LAT source is the uncertainty on the centroid position of the given LAT source, which is typically $\sim 0$. 1 . Most VHE $\gamma$-ray sources are extended, with a typical size of $0.1-0.5$. Let $e$ be the sum of the radius of the $95 \%$ confidence region and the size of the VHE $\gamma$-ray source.

For each LAT source, if a VHE $\gamma$-ray source was found where $d-e<0$, the source pair is classified as a spatially coincident case (i.e. category $Y$ - yes). Given the possible extended nature of many LAT bright sources, a category $P$ (for possible) is defined for pairs where $0<d-e<0.3^{\circ}$, so that the sources seen by LAT and the VHE instruments may actually overlap, and are possibly coincident cases. If no reported VHE $\gamma$-ray source was found with $d-e<0.3^{\circ}$, the LAT source falls into the coincidence level $N$ (for no), i.e., no coincidence with any VHE $\gamma$-ray source. If there are several nearby VHE $\gamma$-ray sources, only the closest VHE $\gamma$-ray source would be considered.

\subsection{Spatial coincidence GeV/TeV pairs}

In the search, 24 coincident cases ( $Y$, among them two are coincident with MILAGRO source only) and 7 possibly-coincident

\footnotetext{
1 Possibly associated with SNR or PWN, but the emission may come from unknown pulsars.

2 For example, HESS J1833-105 (Djannati-Atai et al. 2008b) with only a significance of $4.0 \sigma$ but included in Abdo et al. (2010) as a counterpart of LAT pulsar PSR J1833-1034, is not considered here.
} 
P. H. T. Tam et al.: A search for VHE counterparts of Galactic Fermi bright sources and MeV to TeV spectral characterization

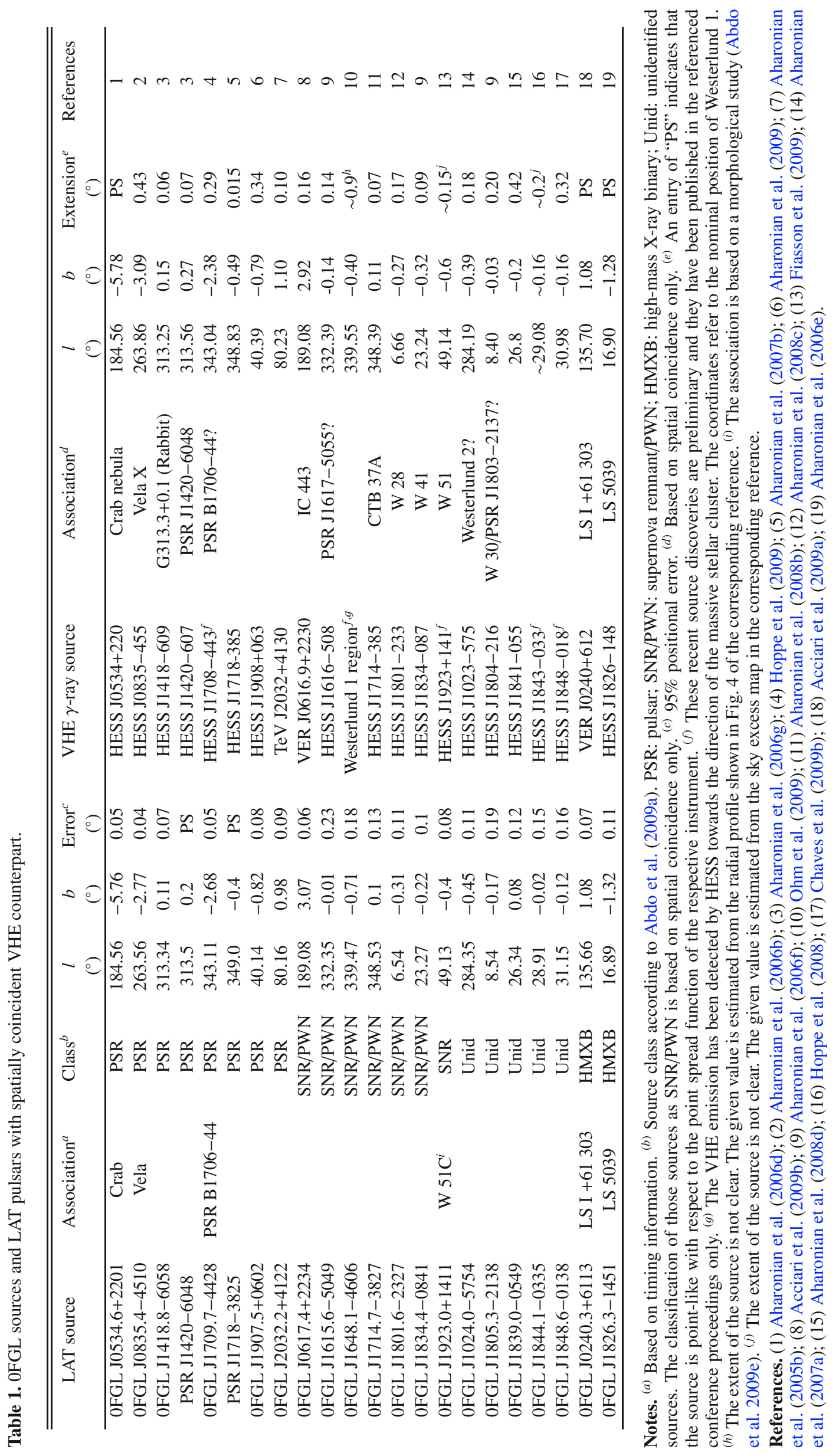


Table 2. OFGL sources with coincident MILAGRO source, but without plausible coincident reported VHE $\gamma$-ray sources. See Table 1 for the nomenclature.

\begin{tabular}{lccccccccc}
\hline \hline LAT source & Class & $\begin{array}{c}l \\
\left({ }^{\circ}\right)\end{array}$ & $\begin{array}{c}b \\
\left({ }^{\circ}\right)\end{array}$ & $\begin{array}{c}\text { Error } \\
\left({ }^{\circ}\right)\end{array}$ & $\begin{array}{c}\text { MILAGRO source } \\
\left({ }^{\circ}\right)\end{array}$ & $\begin{array}{c}l \\
\left({ }^{\circ}\right)\end{array}$ & $\begin{array}{c}b \\
\left({ }^{\circ}\right)\end{array}$ & Extension & References \\
\hline 0FGL J0634.0+1745 & PSR & 195.16 & 4.29 & 0.04 & MGRO C3 & 195.3 & 3.8 & 1.3 & Abdo et al. (2009g) \\
0FGL J2020.8+3649 & PSR & 75.182 & 0.131 & 0.060 & MGRO J2019+37 & 74.8 & 0.4 & $\sim 0.1$ & Abdo et al. (2009g) \\
\hline
\end{tabular}

Table 3. OFGL sources with a possibly coincident VHE $\gamma$-ray source. See Table 1 for the nomenclature.

\begin{tabular}{|c|c|c|c|c|c|c|c|c|c|c|}
\hline LAT source & Class & $\begin{array}{c}l \\
\left({ }^{\circ}\right)\end{array}$ & $\begin{array}{c}b \\
\left({ }^{\circ}\right)\end{array}$ & $\begin{array}{c}\text { Error } \\
\left({ }^{\circ}\right)\end{array}$ & VHE $\gamma$-ray source & Association & $\begin{array}{c}l \\
\left(^{\circ}\right)\end{array}$ & $\begin{array}{l}b \\
\left({ }^{\circ}\right)\end{array}$ & $\begin{array}{c}\text { Extension } \\
\left({ }^{\circ}\right)\end{array}$ & References \\
\hline 0FGL J1814.3-1739 & SNR/PWN & 13.05 & -0.09 & 0.19 & HESS J1813-178 & G12.8-0.2/AX J1813-178 & 12.81 & -0.03 & 0.04 & 1 \\
\hline 0FGL J1634.9-4737 & Unid & 336.84 & -0.03 & 0.08 & HESS J1634-472 & & 337.11 & 0.22 & 0.11 & 1 \\
\hline 0FGL J1741.4-3046 & Unid & 357.96 & -0.19 & 0.2 & HESS J1741-302 ${ }^{a}$ & & 358.4 & 0.01 & ? & 2 \\
\hline 0FGL J1746.0-2900 & Unid & 359.99 & -0.11 & 0.07 & HESS J1745-290 & Sgr A*/G359.95-0.04 & 359.94 & -0.04 & PS & 3 \\
\hline 0FGL J1836.1-0727 & Unid & 24.56 & -0.03 & 0.22 & HESS J1837-069 & & 25.18 & -0.12 & $7.2 \times 3^{\prime}$ & 1 \\
\hline OFGL J2021.5+4026 & PSR & 78.23 & 2.07 & 0.05 & VER J2019+407 ${ }^{a}$ & $\gamma$ Cygni SNR? & 78.33 & 2.54 & $0.16 \times 0.11$ & 4 \\
\hline OFGL J2229.0+6114 & PSR & 106.64 & 2.96 & 0.08 & VER J2227+608 & & 106.35 & 2.71 & $0.27 \times 0.18$ & 5 \\
\hline
\end{tabular}

Notes. ${ }^{(a)}$ These recent source discoveries are preliminary and they have been published in the referenced conference proceedings only.

References. (1) Aharonian et al. (2006f); (2) Tibolla et al. (2009b); (3) Acero et al. (2010); (4) Weinstein et al. (2009); (5) Acciari et al. (2009c).

cases $(P)$ are found. The results are presented in Tables $1-3$. No reported VHE $\gamma$-ray sources are found in the remaining 68 sources.

According to the nature of the LAT sources, the results are summarized as follows:

1. Eight LAT pulsars are spatially coincident with a source detected using IACTs, which may be the VHE-emitting PWN. There are two additional pulsars that are possibly coincident with an IACT source. Two others have a MILAGRO counterpart, but have not yet been detected by IACTs.

2. Among the 13 SNR/PWN candidates in the Fermi BSL, more than half (7) are spatially coincident with a VHE $\gamma$-ray source, and another one is a possibly coincident case. The seemingly high fraction of coincidence is partly due to better coverage of the inner Galaxy region, where most SNR/PWN candidates are located. This results in a generally better sensitivity for this class of sources than for other classes.

3. The two HMXBs listed in the BSL (0FGL J0240.3+6113/ LS I +61 303 and 0FGL J1826.3-1451/LS 5039) are both found to be spatially coincident with a VHE $\gamma$-ray source, identified with the same corresponding binary.

4. Five of the 37 unidentified OFGL sources are spatially coincident with a VHE $\gamma$-ray source. The number increases to nine if possibly coincident cases are included.

In addition, we are aware that a new VHE source near PSR J1119-6127 was announced in an oral presentation ${ }^{3}$, but it has not been published with any written reference to our knowledge. Given that the best-fit centroid and extension were not given, we do not treat it as an entry in our sample ${ }^{4}$.

With such a large number of coincident cases, the relationship between the $\mathrm{GeV}$ and $\mathrm{TeV}$ sources is explored. In the next

\footnotetext{
${ }^{3}$ See http://cxc.harvard.edu/cdo/snr09/pres/ DjannatiAtai_Arache_v2.pdf

${ }^{4}$ In the first LAT catalog, that can be found at http://fermi. gsfc.nasa.gov/ssc/data/access/lat/1yr_catalog/, the authors claim that there is an association of the LAT source 1FGL J1119.4-06127 with a VHE source, designated HESS J1119-614, which may be the same VHE source.
}

section, the $\gamma$-ray spectral energy distributions (SEDs) are constructed for those coincident and possibly coincident $\mathrm{GeV} / \mathrm{TeV}$ source pairs with published VHE spectrum.

\section{The $\gamma$-ray spectral energy distributions}

\subsection{Construction of power-law spectrum in the LAT energy range}

Abdo et al. (2009a) provide photon fluxes and respective errors in two energy bands: low energy $(100 \mathrm{MeV}-1 \mathrm{GeV})$ and high energy $(1-100 \mathrm{GeV})$. Since photon spectra are not given in the BSL, we attempt to estimate the spectra of individual sources.

Assuming that a single pure power law represents the spectrum in the LAT energy range, the photon flux in the low $\left(10^{2}-\right.$ $\left.10^{3} \mathrm{MeV}\right)$ and high energy $\left(10^{3}-10^{5} \mathrm{MeV}\right)$ bands, respectively, are given by $F_{23}=k \int_{0.1}^{1} E^{-\Gamma} \mathrm{d} E$ and $F_{35}=k \int_{1}^{100} E^{-\Gamma} \mathrm{d} E$, where $E$ is measured in GeV, $\Gamma$ is the photon index, and $k$ the normalization at $1 \mathrm{GeV}$. Both $k$ and $\Gamma$ can be calculated from these two expressions. Using the available flux errors $\left(\Delta F_{23}\right.$ and $\left.\Delta F_{35}\right)$, uncertainties in $k$ and $\Gamma(\Delta k$ and $\Delta \Gamma)$ are obtained by error propagation. The spectra are then constructed in the form of "bowties". For those where $F_{23}$ is given as a 2- $\sigma$ upper limit, the calculated $\Gamma$ can be treated as an upper limit, and the reconstructed spectra can be seen as the "softest possible" power-law spectra. The power-law spectra are plotted from $100 \mathrm{MeV}$ up to a certain maximum energy, $E_{\max }(\leq 100 \mathrm{GeV})$, which is defined by requiring that the photon spectrum above $E_{\max }$ contains 10 photons over the three months of observations ${ }^{5}$. This results in a range of values for $E_{\max }$ from $\sim 3 \mathrm{GeV}$ to $100 \mathrm{GeV}$. The single power-law assumption does not hold in general. Given the limited information available in the BSL, such an assumption should be seen as a very rough estimation of the source spectra and it is used in this work for a visual $\mathrm{GeV} / \mathrm{TeV}$ spectral comparison. A cut-off between the $\mathrm{GeV}$ and $\mathrm{TeV}$ bands has been measured for several sources including pulsars. Therefore, we also plot the best-fit spectra when a detailed LAT spectrum is available in the literature (Vela, Crab, Geminga, PSR J1706-44,

\footnotetext{
${ }_{5}$ Using the LAT on-axis effective area above $1 \mathrm{GeV}$ of $\sim 8000 \mathrm{~cm}^{2}$ and a mean on-axis exposure of $\sim 1 \mathrm{Ms}$ (Abdo et al. 2009a).
} 
0FGLJ0534.6+2201

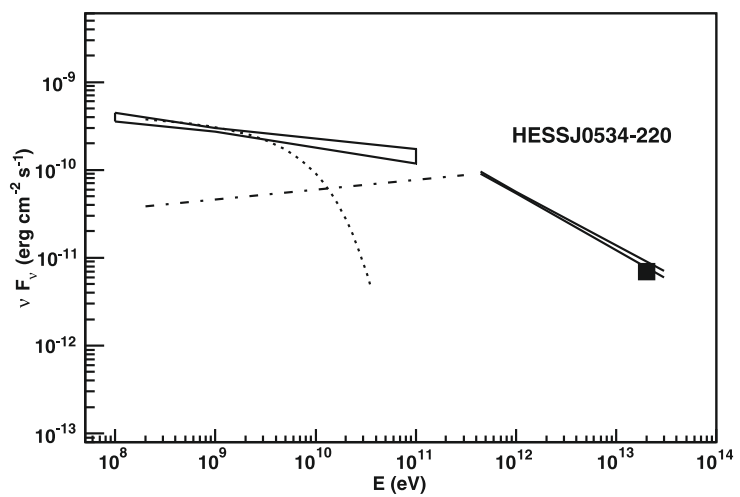

OFGLJ0835.4-4510

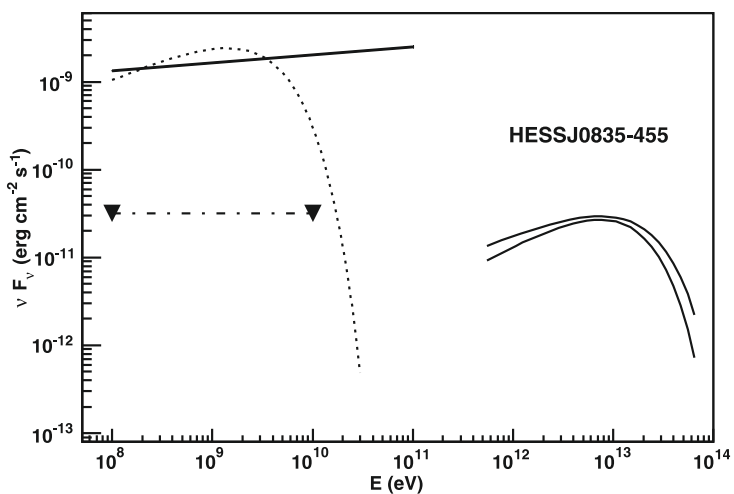

0FGLJ0634.0+1745

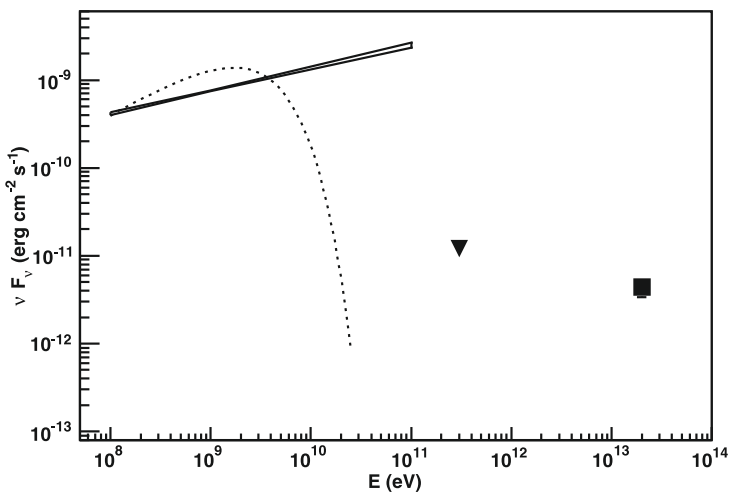

OFGLJ1709.7-4428

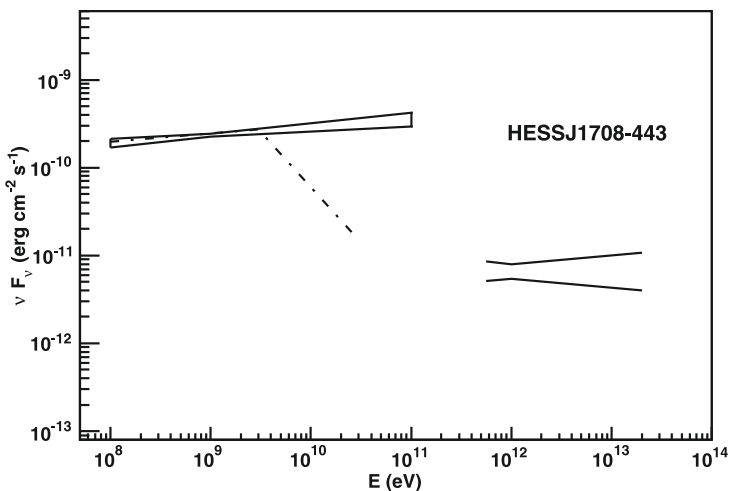

Fig. 1. MeV to TeV spectra of four EGRET pulsars and their proposed nebulae. Upper left: Crab (OFGL J0534.6+2201). The pulsar (dotted line) and nebula (dashed-dotted line) spectral components are those reported in Grondin et al. (2009). The VHE spectra are taken from Aharonian et al. (2006d), and the MILAGRO measurement at $20 \mathrm{TeV}$ is shown (Abdo et al. 2007). Upper right: Geminga (0FGL J0634.0+1745). The pulsar spectrum (dotted line) is the one reported in Celik et al. (2009). The triangle denotes the upper limit reported in Finnegan et al. (2009), and the MILAGRO measurement at $20 \mathrm{TeV}$ is also indicated (Abdo et al. 2007). Lower left: Vela (0FGL J0835.4-4510). The dotted line represents the Vela spectrum as shown in Abdo et al. (2009c), while the nebula component is constrained by the two triangles joined by the dashed-dotted line. The curved VHE spectrum is taken from Aharonian et al. (2006b). Lower right: PSR B1706-44 (0FGL J1709.7-4428). The dashed-dotted line denotes the two power-law model spectra derived in Gargano et al. (2009). Both LAT energy spectra (though different above $3 \mathrm{GeV}$ ) are consistent with the photon flux in the 1-100 GeV band of this source (Abdo et al. 2009a). The VHE spectrum is taken from Hoppe et al. (2009).

and LS I +61 303). For the cases of 0FGL J0617.4+2234 and OFGL J1746.0-2900, the double power-law spectra derived for 3EG J0617+2238 and 3EG J1746-2851, respectively, by Bertsch et al. (2000) are also shown for comparison.

\subsection{The MeV-TeV SEDS}

The sources considered here are those 0FGL/VHE pairs with spatial coincidence levels $Y$ and $P$ and with VHE spectral information available in the literature. For HESS J1923+141 where only a VHE flux is given, a typical spectral index is assumed. In addition, there are two pulsars for which a MILAGRO candidate counterpart is reported but there is no VHE $\gamma$-ray detection using IACTs (see Table 2).

The SEDs of the 28 cases in the energy range from $100 \mathrm{MeV}$ to $>1 \mathrm{TeV}$ are depicted in Figs. 1 to 9. Systematic errors in spectral indices and normalization are not shown, which for $\mathrm{TeV}$ spectra are $\sim 20 \%$ for most sources and for $\mathrm{GeV}$ spectra are 20-30\% (the latter inferred from flux estimation systematics in Abdo et al. 2009a). An overall inspection of the SEDs immediately shows that single power laws from $200 \mathrm{MeV}$ to $\sim 10 \mathrm{TeV}$ cannot describe most GeV-TeV $\gamma$-ray spectra. This is not surprising given the large range in photon energy (i.e. five orders of magnitude), as no photon spectrum from any emission mechanism is expected to be unbroken for such a broad energy span.
The only example for which a pure power law may still work is 0FGL J1836.1-0727/HESS J1837-069, which is a possibly coincident pair $(P)$. The most common board-band behaviors are a cut-off at energies below $\sim 100 \mathrm{GeV}$ (dominating in the pulsar class) and a spectral break between the LAT and the VHE bands (dominating in the unidentified LAT sources).

The SEDs of the LAT source classes including pulsars, SNR/PWN candidates, and unidentified $\gamma$-ray sources are presented in this section. LS I +61 303 and the Galactic center region are discussed in Sects. 4.5 and 4.6, respectively.

\subsubsection{Pulsars}

Figure 1 shows the four $\gamma$-ray pulsars known in the EGRET era, Fig. 2 shows the four radio pulsars first detected in $\gamma$-rays by LAT, and Fig. 3 shows the three new pulsars after a blind search for pulsations in the LAT data (Abdo et al. 2009b). Besides the Crab, no off-pulse emission is found in the LAT data of the other 10 pulsars, suggesting that most of the emission from pulsars seen with LAT is pulsed and comes from the pulsars themselves. On the other hand, extended regions are seen at energies above $100 \mathrm{GeV}$ in these 10 cases (except for the Crab, which appears point-like to all IACTs). Their VHE emission $(>100 \mathrm{GeV})$ is unpulsed, and for many of them (e.g. Vela X) this emission have been attributed to PWNe, although in some cases other 


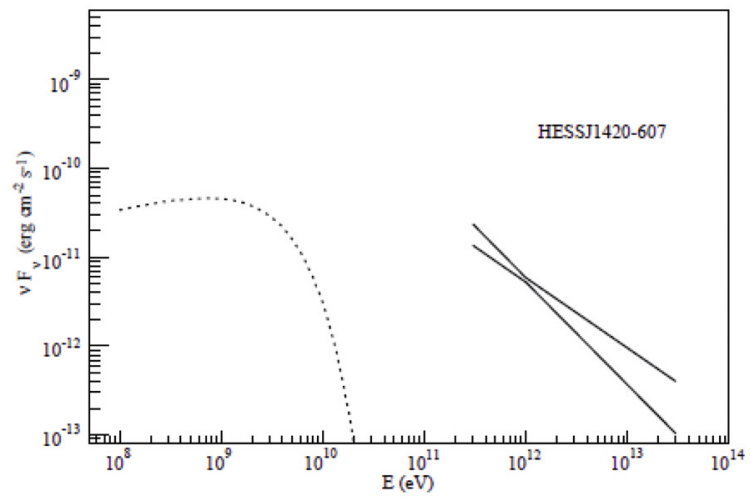

0FGLJ2020.8+3649

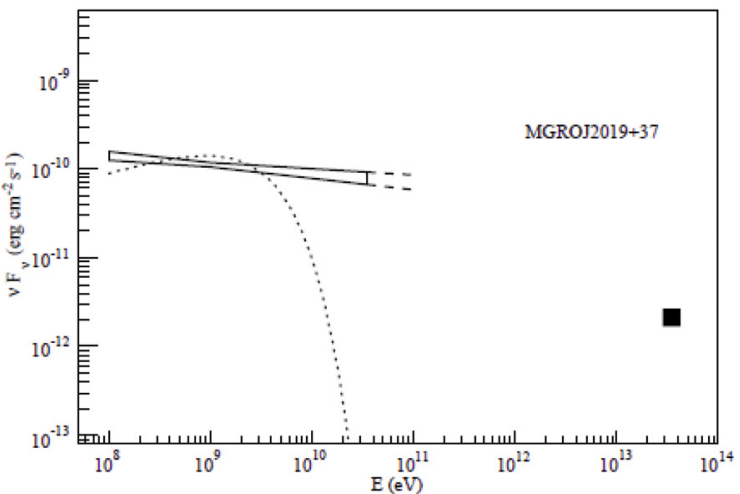

PSRJ1718-3825

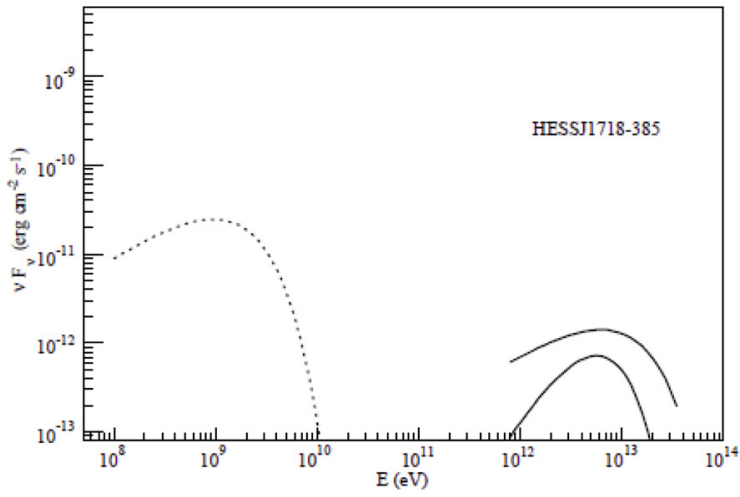

FGLJ2229.0+6114

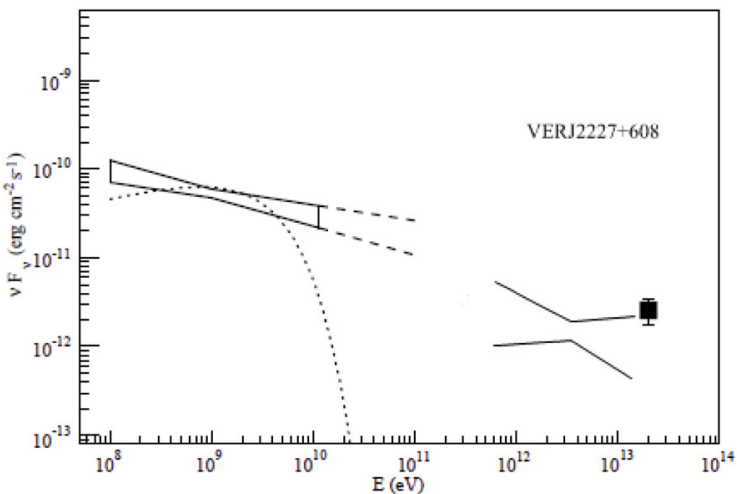

Fig. 2. MeV to TeV spectra of the four radio pulsars first detected in $\gamma$-rays by LAT. The spectra below $300 \mathrm{GeV}$ are taken from Abdo et al. (2010). Upper left: The VHE spectrum is taken from Aharonian et al. (2006g). Upper right: The VHE spectrum presented in Aharonian et al. (2007b) is shown. The two curves represent the upper and lower limits of the spectrum, taking measurement errors into account. Lower left: The flux at $35 \mathrm{TeV}$ is taken from Abdo et al. (2009g). Lower right: The flux at $20 \mathrm{TeV}$ is taken from Abdo et al. (2007) and the VHE spectrum is taken from Acciari et al. (2009c).

possibilities exist to explain the VHE $\gamma$-ray source (e.g. a spatially coincident SNR).

The SEDs of the pulsars mainly depict the pulsed component in the LAT energy band and the unpulsed component in the VHE band. Previous observations suggest that the emission below and above $\sim 100 \mathrm{GeV}$ mainly comes from two different emission regions, e.g. pulsed emission from the pulsar magnetosphere and unpulsed emission from the PWN. It can be seen that (1) a cut-off must exist between the LAT "bowties" and the corresponding VHE spectra (with the notable exception of the $\mathrm{Crab}$ ), which is demonstrated with a detailed spectral study of pulsars (e.g., Abdo et al. 2010); (2) the energy output at GeV energies is at least an order of magnitude higher than in the VHE band. This indicates that, for the pulsar population presented in this section, the PWNe radiate less energy than the $\gamma$-ray pulse emitting regions.

However, the power-law derived LAT spectra are not always good representations of the reported spectra for individual sources. This is demonstrated in Fig. 1 where both the "bowtie" spectra and the derived spectra in Abdo et al. (2010) are shown. In all the other cases, only the spectra as presented in Abdo et al. (2010) are depicted.

\subsubsection{SNR/PWN candidates}

The SEDs of those OFGL sources classified as SNR/PWN candidates are shown in Figs. 4 and 5. The GeV-TeV spectral connection varies among the sources in this class. The $\mathrm{TeV}$ spectra are not simply the power-law tails of the GeV spectra. There are cases where the extrapolation of the LAT "bowtie" to $\mathrm{TeV}$ energies is at least an order of magnitude higher than the measured VHE flux level (e.g. the spatially coincident case 0FGL J1801.6-2327/HESS J1801-233, a cut-off occurs between the two energy bands), while for another coincident case (0FGL J1834.4-0841/HESS J1834-087), the power-law extrapolation to the VHE band is below the measured VHE level and a second spectral component above $\sim 200 \mathrm{GeV}$ is needed to explain the $\mathrm{TeV}$ excess.

There is only one case (0FGL J0617.4+2234) where a broken power law describes the LAT spectrum better than a single power law. The "bowties", which are derived a priori from power laws, may be closer to the real spectra compared to the case of pulsars. If that is the case for 0FGL J1801.6-2327/HESS J1801233 and 0FGL J1923.0+1411/HESS J1923+141, a spectral break may occur at energies in the largely unexplored energy range of $10-100 \mathrm{GeV}$ for these two sources ${ }^{6}$.

\subsubsection{Unidentified LAT sources}

The SEDs of those OFGL sources without obvious counterparts are shown in Figs. 6 and 7. There is so far no published

\footnotetext{
6 The LAT spectrum for OFGL J1801.6-2327 is the softest possible power law, while the HESS J1923+141 spectrum is derived assuming a power-law index $\Gamma=2.8$.
} 


\section{FGLJ1418.8-6058}

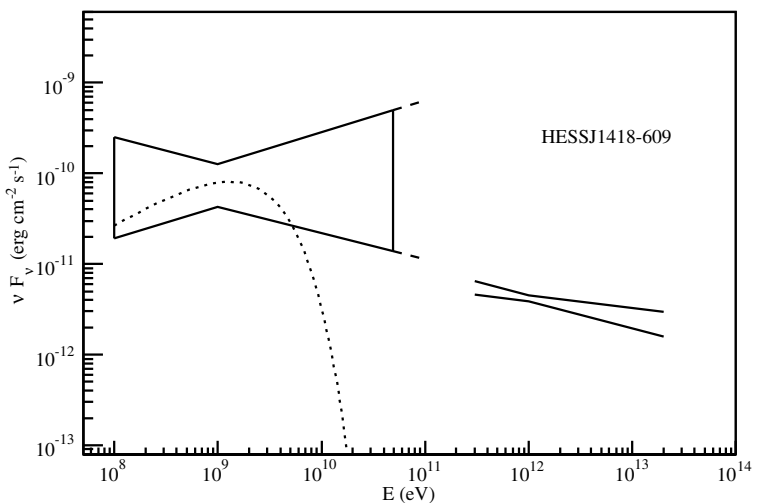

OFGLJ2032.2+4122

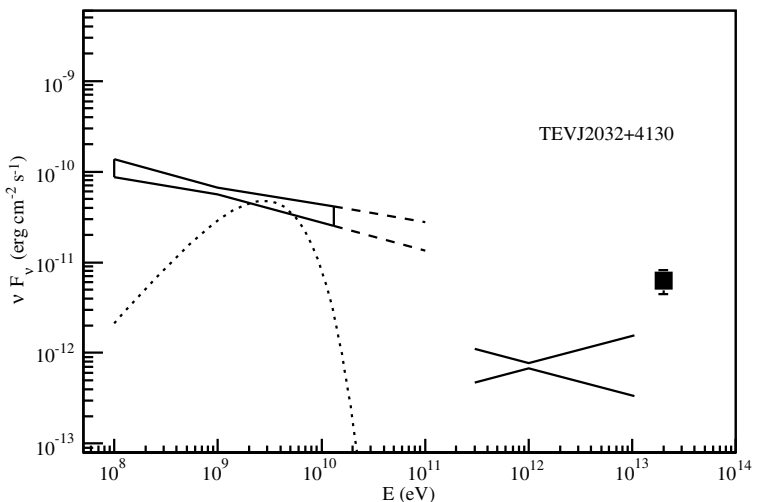

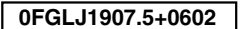

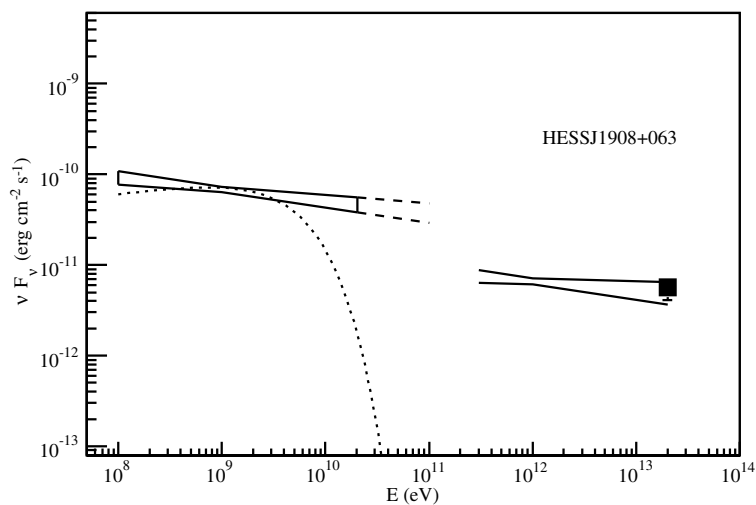

Fig. 3. MeV to TeV spectra of the three new pulsars discovered in a blind search for pulsation in the LAT data. The spectra below $300 \mathrm{GeV}$ are taken from Abdo et al. (2010). Upper left: The VHE spectrum is taken from Aharonian et al. (2006g). Upper right: The VHE spectrum presented in Djannati-Atai et al. (2008a) is shown, together with the coincident MILAGRO source flux at 20 TeV (Abdo et al. 2007). Lower left: The VHE spectrum is the one presented in Aharonian et al. (2005b), while the MILAGRO flux at 20 TeV is also shown (Abdo et al. 2007).

spectra of this LAT source class. For the case of 0FGL J1839.0-0549/HESS J1841-055, the spectrum may span from $\sim 100 \mathrm{MeV}$ to $\sim 80 \mathrm{TeV}$, with a possible break within or close to the "energy gap" at $\sim 60-500 \mathrm{GeV}$. If the GeV and $\mathrm{TeV}$ sources are indeed associated, they might represent a group of "dark accelerators" which have a broad $\gamma$-ray spectrum. All SEDs are consistent with the assumption that a spectral break exists between the two energy bands, except for the case of 0FGL J1805.3-2138/HESS J1804-216, a spatially coincident case $(Y)$

\subsection{Comparison of the flux and photon indices in the GeV and TeV energy bands}

A comparison of the flux levels in the $\mathrm{GeV}$ and $\mathrm{TeV}$ energy bands for coincident $\mathrm{GeV} / \mathrm{TeV}$ sources (category $Y$ ) is depicted. Figure 10 shows the photon flux in the $100 \mathrm{MeV}-1 \mathrm{GeV}$ band plotted against the one in the $1-10 \mathrm{TeV}$ band (derived according to Sect. 3.1). For most sources, the photon flux in the $1-10 \mathrm{TeV}$ band, $F_{1-10 \mathrm{TeV}}$, is about $10^{-5}$ to $10^{-6}$ the flux in the $0.1-1 \mathrm{GeV}$ band. Figure 11 shows the photon flux in the $1-100 \mathrm{GeV}$ band plotted against that in the $1-10 \mathrm{TeV}$ band. For most sources, photon flux in the $1-10 \mathrm{TeV}$ band, $F_{1-10 \mathrm{TeV}}$, is about $10^{-4}$ to $10^{-5}$ of that in the $1-100 \mathrm{GeV}$ band.

Figure 12 depicts the photon indices in the $0.1-100 \mathrm{GeV}$ band derived according to Sect. 3.1 against the photon index in the $1-10 \mathrm{TeV}$ band. It can be seen that the $\mathrm{TeV}$ spectra are similar to or harder than the $\mathrm{GeV}$ spectra for most sources, i.e. $0 \lesssim\left(\Gamma_{1-10 \mathrm{TeV}}-\Gamma_{0.1-100 \mathrm{GeV}}\right) \lesssim 1$.

\section{Notes on selected sources}

Although detailed analysis of the LAT data for each individual source is beyond the scope of this paper, some comments on the following sources are given.

\subsection{Crab pulsar and nebula}

The Crab pulsar and nebula are among the best-studied nonthermal objects in the sky. The pulsed emission above $100 \mathrm{MeV}$ and up to $\sim 10 \mathrm{GeV}$ is clearly detected with LAT. Two strong peaks are seen in the phase histogram. A spectral fit of the pulsed emission using a power law with an exponential cut-off gives a cut-off energy of $\sim 8.8 \mathrm{GeV}$ (Grondin et al. 2009). There is evidence of pulsed emission up to $\sim 25 \mathrm{GeV}$, as measured using the MAGIC telescope (see Fig. 1, Aliu et al. 2008). The flux reported by MAGIC is consistent with the exponential cut-off in the spectrum measured by LAT.

Evidence of unpulsed emission was already present in the EGRET data (de Jager et al. 1996). The LAT measurement of this component can be well fit by a single power law with $\Gamma \sim$ 1.9 up to $\sim 300 \mathrm{GeV}$. This unpulsed spectrum agrees well with the VHE spectra measured by the IACTs MAGIC, HESS, and VERITAS (Grondin et al. 2009). In particular, there appears to 

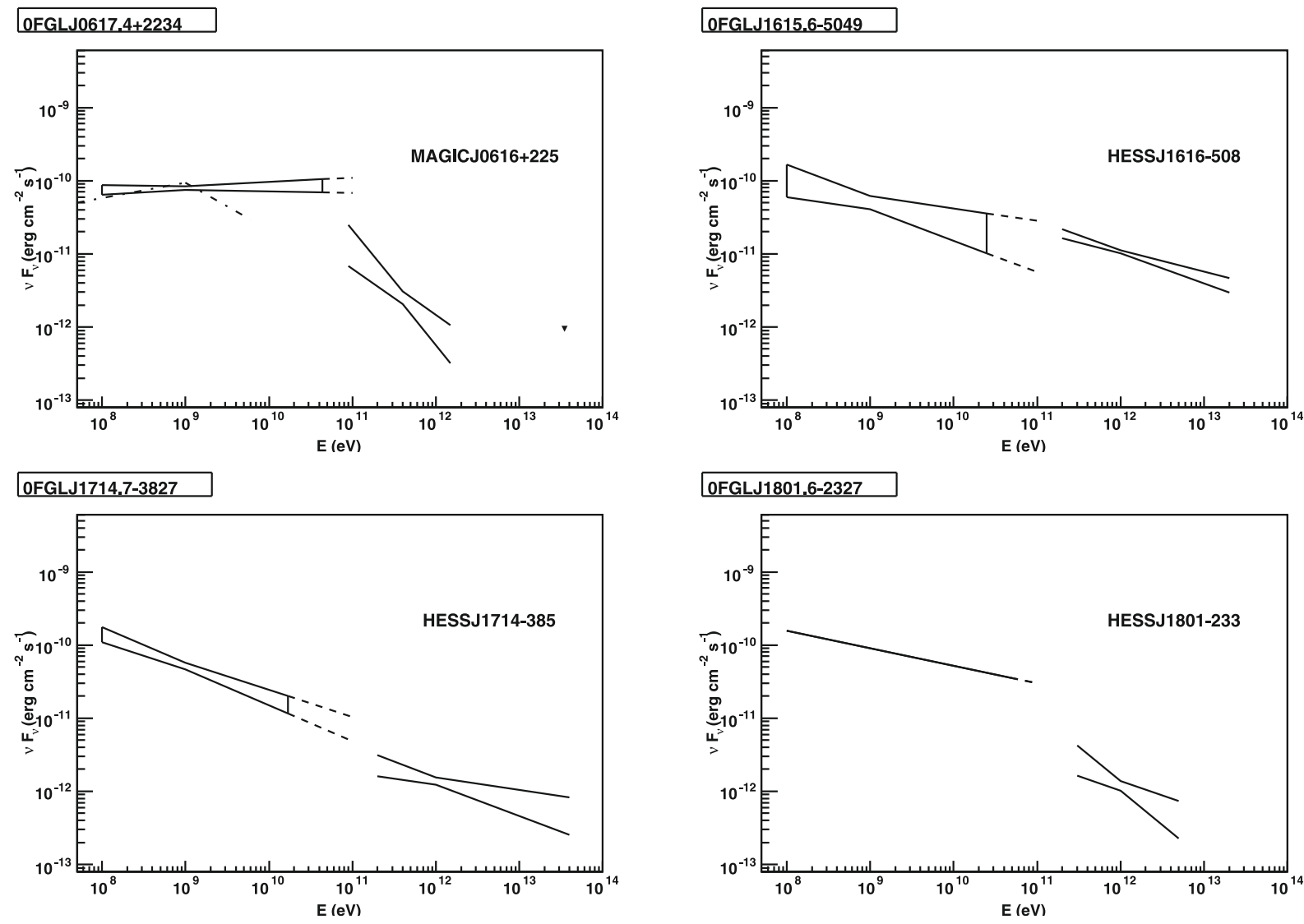

Fig. 4. $\mathrm{MeV}$ to $\mathrm{TeV}$ spectra of four SNR/PWN candidate 0FGL sources. Spectra at $>100 \mathrm{GeV}$ energies are taken from Albert et al. (2007) (MAGIC J0616+225), Aharonian et al. (2006f) (HESS J1616-508), Aharonian et al. (2008b) (HESS J1714-385), and Aharonian et al. (2008c) (HESS J1801-233). The broken power-law spectrum (dashed-dotted line) derived for 3EG J0617+2238 is taken from Bertsch et al. (2000). The flux at $35 \mathrm{TeV}$ at the position of 0FGL J0617.4+2234 is that given in Abdo et al. (2009g).

be a deviation from a pure power law in the MAGIC spectrum below $\sim 100 \mathrm{GeV}$ (Albert et al. 2008).

\subsection{Vela pulsar and Vela $X$}

The Vela pulsar is the strongest persistent GeV source and was the first target of LAT observations. The complex pulse profile is dominated by two peaks with a pronounced "bridge" between them. The phase-averaged spectrum, which is essentially the pulsed emission, can be described well by a power law with an exponential cut-off at $\sim 2.9 \mathrm{GeV}$. The off-pulse emission is much weaker, and a $95 \%$ C.L. upper limit of the photon flux of $1.8 \times 10^{-7} \mathrm{~cm}^{-2} \mathrm{~s}^{-1}$ is derived at the pulsar position in the 0.1-10 GeV band (shown in Fig. 1, Abdo et al. 2009c).

To the south of the pulsar, an extended VHE $\gamma$-ray source spatially coincident with the Vela X region, HESS J0835-455, has been detected. The observations represent the first measurement of an SED peak in a VHE $\gamma$-ray source (Aharonian et al. 2006b). The power law with exponential cut-off fit of this PWN is reproduced in Fig. 1. An analysis of the Vela $\mathrm{X}$ region does not establish a nebula component based on the first three months of LAT observations (Lemoine-Goumard et al. 2009).

\subsection{Geminga}

The Geminga pulsar is the first known radio-quiet $\gamma$-ray pulsar in the sky (Bertsch et al. 1992). See Fig. 1 for its SED. While EGRET data are well fit by a single power law up to $2 \mathrm{GeV}$ (but shows evidence of a cut-off above $2 \mathrm{GeV}$; Mayer-Hasselwander et al. 1994), the cut-off energy is determined to be $\sim 2.6 \mathrm{GeV}$ using the first seven months of LAT data (Celik et al. 2009). There appears to be an excess at $\sim 20 \mathrm{GeV}$ when compared to the fit with a power law with exponential cut-off. The reason may be the low statistics or effects of the fitting method, but it might also indicate a separate and harder spectral component (Celik et al. 2009). There is as yet no evidence of unpulsed emission.

Evidence of multi-TeV emission around the pulsar was reported in the MILAGRO survey of the Galactic plane (Abdo et al. 2007) and in the search for MILAGRO counterparts of Fermi sources (Abdo et al. 2009g), using a point source analysis at $\sim 3 \sigma$ (post-trial) significance levels. Assuming that the emission is extended, the significance increases to $6.3 \sigma$ at the position of the pulsar. If the detection is real, the size of the MILAGRO emission is $\sim 2.6$. At a distance of only $\sim 250 \mathrm{pc}$, this extent is similar to more distant PWN (Abdo et al. 2009g). On the other hand, VERITAS observations resulted in no detection but rather a $99 \%$ C.L. flux upper limit (above $300 \mathrm{GeV}$ ) of $2 \times 10^{-12} \mathrm{~cm}^{-2} \mathrm{~s}^{-1}$, assuming point source emission from the pulsar (Finnegan et al. 2009). Although IACTs suffer from reduced sensitivity when observing very extended source (which scales as $\theta^{-1}$ with $\theta$ being the extension), observations of Geminga with IACTs are crucial for verifying the MILAGRO claim and helping us to understand the $\gamma$-ray emission from Geminga.

\subsection{PSR B1706-44}

Gamma-ray pulsations from PSR B1706-44 were discovered by EGRET; the observations revealed a triple-peaked pulse profile 


\section{OFGLJ1814.3-1739}

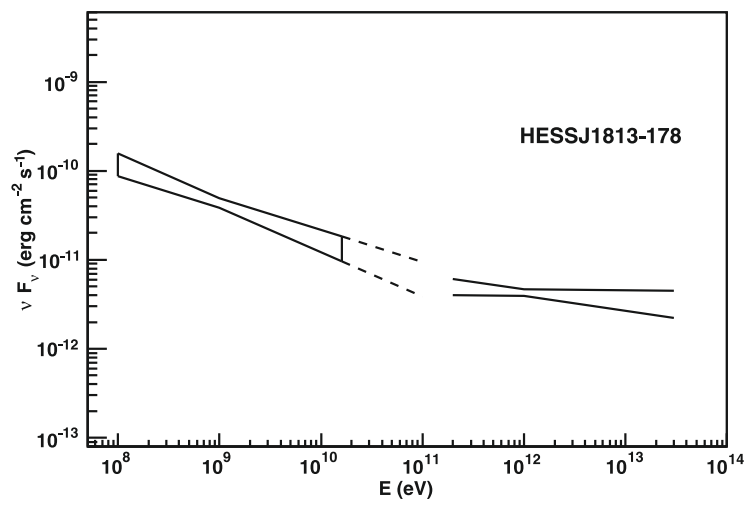

0FGLJ1923.0+1411

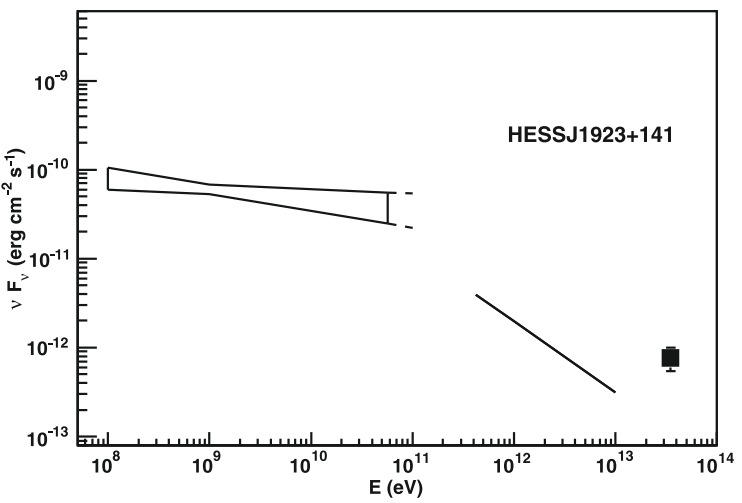

\section{OFGLJ1834.4-0841}

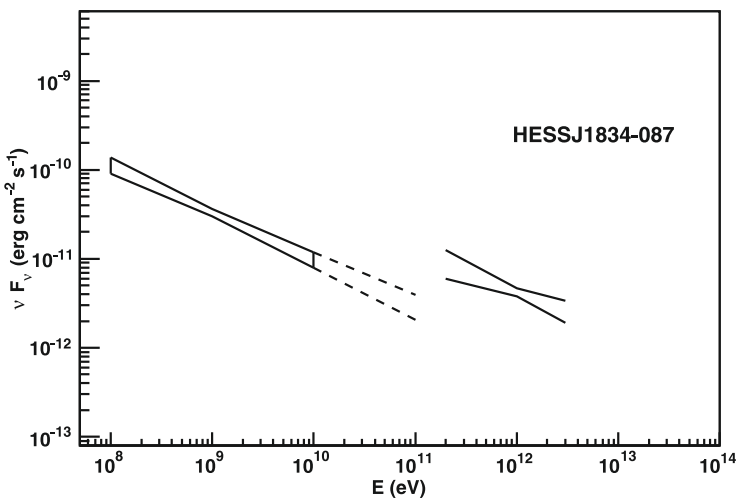

Fig. 5. MeV to TeV spectra of three SNR/PWN candidate 0FGL sources. Spectra at $>100$ GeV energies are taken from Aharonian et al. (2006f) (HESS J1813-178 and HESS J1834-087). For HESS J1923+141, an assumed photon index of 2.8 is used in deriving the spectrum using the flux given in Fiasson et al. (2009), and the flux at $35 \mathrm{TeV}$ is that given in Abdo et al. (2009g). There is evidence of a steepening above several GeV (Abdo et al. 2009e).

but no evidence of unpulsed emission (Thompson et al. 1996). More recently, the pulsar was also detected by Fermi/LAT as the bright source 0FGL J1709.7-4428. The phase-averaged spectra measured by EGRET and LAT are both described well by a broken power law (up to $30 \mathrm{GeV}$, in the case of the LAT spectrum). The break energy measured by LAT is $3 \mathrm{GeV}$, while in deriving the EGRET spectrum, it is fixed at $1 \mathrm{GeV}$ (Thompson et al. 1996; Bertsch et al. 2000). The LAT power-law index steepens from a value of $\sim 1.9$ (below $3 \mathrm{GeV}$ ) to $\sim 3.3$ (above $3 \mathrm{GeV}$ ), as shown in Fig. 1. This spectrum and the power-law spectrum derived using the method described in Sect. 3.1 are both consistent with the photon flux in the $1-100 \mathrm{GeV}$ band reported in Abdo et al. (2009a).

The discovery of an extended source of VHE emission in the vicinity of PSR B1706-44 was recently reported by HESS (Hoppe et al. 2009). The $\mathrm{TeV}$ source is quite hard $(\Gamma \sim 2.0)$, more so than the high-energy part of the pulsar spectrum. The VHE $\gamma$-ray emission might be related to a relic PWN of PSR B1706-44 and/or to the SNR G343.1-2.3 (Hoppe et al. 2009).

\section{5. $L S I+61303$}

LS I +61 303 is the first X-ray binary where periodic $\gamma$-ray emission has been detected at both $\mathrm{GeV}$ (Abdo et al. 2009d) and TeV energies (Acciari et al. 2009a; Albert et al. 2009). Its SED is shown in Fig. 8. The "bowtie" looks nicely connected to the measured VHE spectrum, but a cut-off energy at $\sim 6 \mathrm{GeV}$ is reported (Abdo et al. 2009d). Furthermore, the timing measurements in both the $\mathrm{GeV}$ and $\mathrm{TeV}$ bands show that the maximum emission occurs at different orbital phases, namely, close to periastron for $<100 \mathrm{GeV}$ emission and close to apastron for VHE emission. This suggests different emission mechanisms in the two bands, as noted in Abdo et al. (2009d).

\subsection{Galactic center region}

The Galactic center is among the richest and most complex regions in the Galaxy, owing to the large number of possible sources and the difficulty of correctly modeling the diffuse emission due to cosmic-ray interaction with the local molecular clouds. This problem is extremely relevant at $\mathrm{GeV}$ energies, as demonstrated by EGRET measurements. The discovery of new VHE $\gamma$-ray sources close to the Galactic center is important for studying the role of diffuse Galactic emission versus the emission from resolved sources in this region (Tibolla et al. 2009a).

One GeV source, 0FGL J1746.0-2900, is detected with a significance of $36 \sigma$ in the neighborhood of the Galactic center. The best-fit position for 0FGL J1746.0-2900 is RA = $17^{\mathrm{h}} 46^{\mathrm{m}} 1^{\prime \prime}$. 4 , Dec $=-29^{\circ} 0^{\prime} 18^{\prime \prime}(\mathrm{J} 2000)$ with a 95\% C.L. error radius of $4^{\prime}$ (Abdo et al. 2009a). The HESS Collaboration also reports a detection of a source towards the Galactic center, localized at RA $=17^{\mathrm{h}} 45^{\mathrm{m}} 39^{\prime} \cdot 6 \pm 0 . ' 4$ (stat) $\pm 0 .{ }^{\prime} 4$ (sys), Dec $=-29^{\circ} 0^{\prime} 22^{\prime \prime} \pm 6^{\prime \prime}$ (stat) $\pm 6^{\prime \prime}$ (sys) (J2000; Acero et al. 2010). Based on the procedure described in Sect. 2.2, the OFGL J1746.0-2900/HESS J1745-290 pair falls into the 

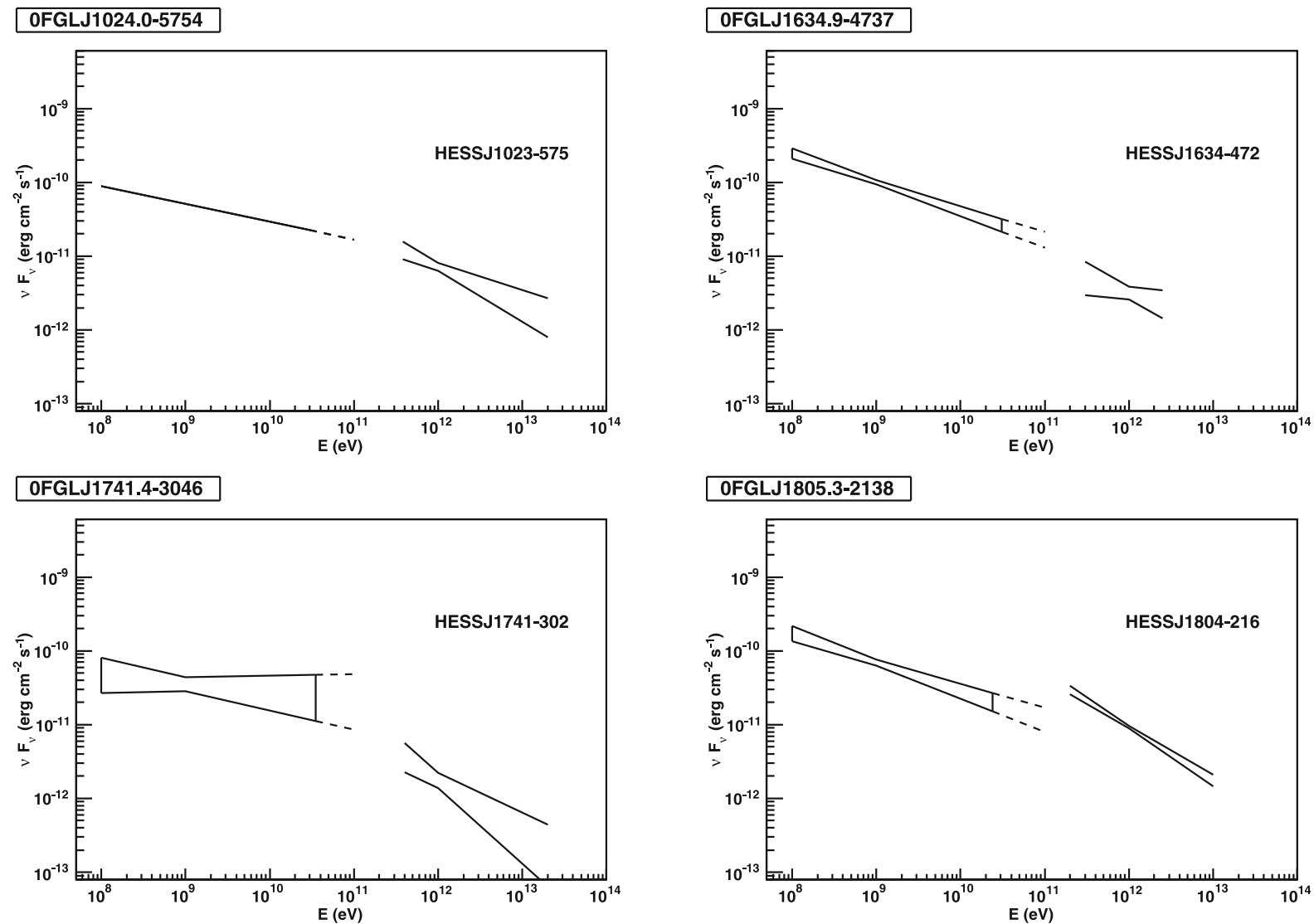

Fig. 6. $\mathrm{MeV}$ to $\mathrm{TeV}$ spectra of four unidentified OFGL sources. Spectra at $>100 \mathrm{GeV}$ energies are taken from Aharonian et al. (2007a) (HESS J1023-575), Aharonian et al. (2006f) (HESS J1634-472 and HESS J1804-216), and Tibolla et al. (2009b) (HESS J1741-302).

category of possibly coincident cases. With better photon statistics, one of the fundamental questions that the LAT can hopefully address is whether the $\mathrm{GeV}$ and $\mathrm{TeV}$ sources are indeed spatially coincident.

The spectra of 0FGL J1746.0-2900 and HESS J1745-290 are shown in Fig. 9. The spectra in the two bands do not appear to be described by a single power law, and there seems to be an order-of-magnitude drop-off in the energy range $\sim 10-100 \mathrm{GeV}$. Although detailed analysis of the LAT data is beyond the scope of this paper, this simple inspection does not indicate that they are from the same emission component (although large uncertainties due to systematics in this region do not permit stronger conclusions at this time). For reference, the broken power-law fit of 3EG J1746-2851 (Bertsch et al. 2000) is also shown in Fig. 9.

\section{Discussion}

In this work, the first comparison of the GeV and VHE $\gamma$-ray sources after the launch of LAT is presented, which takes the advantage of the significantly improved LAT angular resolution and sensitivity compared to EGRET. Below are a list of preliminary results drawn from this work:

1. With the better localization and morphological information of VHE $\gamma$-ray sources compared with OFGL sources, the nature of the LAT sources may be better revealed through their VHE counterparts. Table 1 lists the potential counterparts of some VHE $\gamma$-ray sources that are coincident with
OFGL sources. For example, HESS J1804-216 may be related to $\mathrm{W} 30$, which may help in understanding the nature of the unidentified source 0FGL J1805.3-2138.

2. Results of several LAT-detected pulsars show cut-offs at energies $\sim 1-10 \mathrm{GeV}$, similar to the assessment of Funk et al. (2008) for EGRET-detected pulsar systems. Therefore, a VHE counterpart ( $\sim 0.1-10 \mathrm{TeV})$ of a LAT pulsar most likely represents the associated PWN, with a shell-type supernova as a viable alternative. This is particularly important for those new pulsars discovered by LAT. The VHE counterparts coincident with the six LAT pulsars may be the associated PWN, although other explanations (e.g. shell-type SNR) are also possible. The question of whether typical $\gamma$-ray pulsars are accompanied by VHE-emitting nebulae can be tested by observing them in the VHE domain.

3. Through broad-band $\gamma$-ray spectra of SNRs, one may in principle distinguish between hadronic and leptonic scenarios. A study of RX J1713.7-3946 using five years of simulated LAT observations (Atwood et al. 2009) shows that the energy flux level for the hadronic scenario differs by around a factor of two from that for the leptonic scenario and that a spectral break may be more prominent for the latter. The SNR sample shown in Figs. 4 and 5 do not seem to support either scenario, although it is too early to draw any conclusion based on the three-month 0FGL dataset. If a hadronic scenario is found to be more viable, this would support the current hypothesis that shell-type SNRs are cosmic-ray sources.

4. Previous studies did not reveal a strong correlation between the $\mathrm{GeV} / \mathrm{TeV}$ populations. Reimer et al. (2008) list $16 \mathrm{HESS}$ sources without counterparts from the $3 \mathrm{EG}$ catalog. Among 
P. H. T. Tam et al.: A search for VHE counterparts of Galactic Fermi bright sources and MeV to TeV spectral characterization

\section{OFGLJ1836.1-0727}

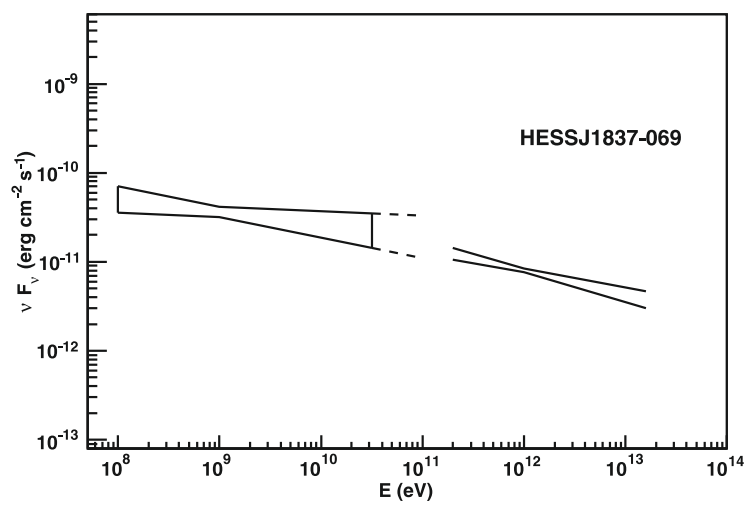

0FGLJ1848.6-0138

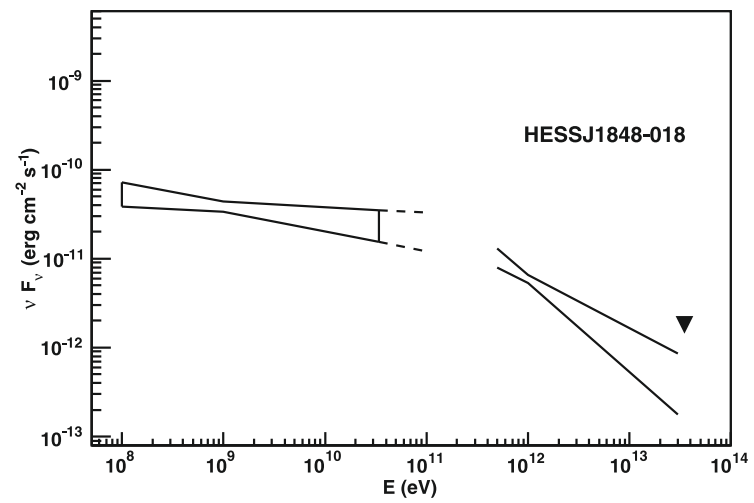

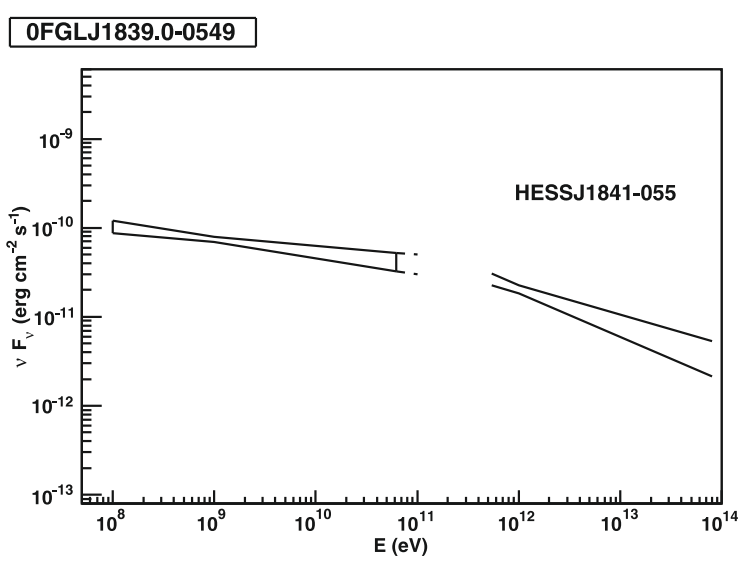

Fig. 7. MeV to TeV spectra of three unidentified OFGL sources. Spectra at >100 GeV energies are taken from Aharonian et al. (2006f) (HESS J1837-069), Aharonian et al. (2008d) (HESS J1841-055), and Chaves et al. (2009b) (HESS J1848-018). The flux at 35 TeV at the position of OFGL J1848.6-0138 is the one given in Abdo et al. (2009g).

0FGLJ0240.3+6113

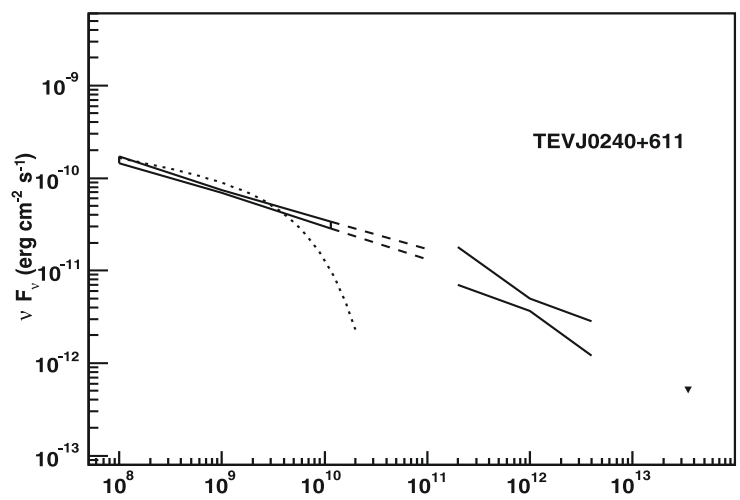

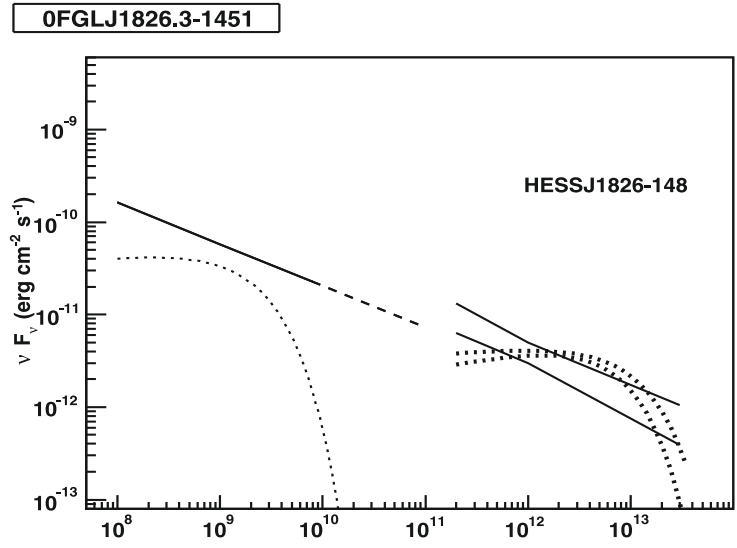

Fig. 8. $\mathrm{MeV}$ to TeV spectra of the two X-ray binaries in the OFGL catalog. The phase-averaged exponential cut-off spectrum in the GeV range of LS I +61 303 (left) is taken from Abdo et al. (2009d). That of LS 5039 is taken from Abdo et al. (2009f). Spectra at $>100 \mathrm{GeV}$ energies are taken from Albert et al. (2009) (for a partial phase of LS I +61 303 during which VHE emission is detected) and Aharonian et al. (2006e) (for two phases of LS 5039). The flux at $35 \mathrm{TeV}$ for LS I +61 303 is that given in Abdo et al. (2009g).

them, new associations are found in the present study and are presented in Table 4, thanks mostly to the discovery of new $\mathrm{GeV}$ sources with LAT. Reimer et al. (2008) also present 11 sources in the $3 \mathrm{EG}$ catalog without HESS counterpart. Among them, 0FGL J1709.7-4428 (the 0FGL counterpart of 3EG J1710-4439; Abdo et al. 2009a) is now found to be associated with HESS J1708-443, a source discovery reported in Hoppe et al. (2009).

5. All spatially coincident $\mathrm{GeV}$ and $\mathrm{TeV}$ pairs during the EGRET era are essentially consistent with one single spectral component (see Figs. 4-6 in Funk et al. 2008). With the significantly enhanced sensitivity of LAT, new relations between the $\mathrm{GeV}$ and $\mathrm{TeV}$ spectra are apparent in the SEDs. The SNR candidate 0FGL J1834.4-0841 and the unidentified OFGL J1805.3-2138 (and their likely VHE counterparts) represent the first examples for which the $\mathrm{GeV} / \mathrm{TeV}$ spectrum cannot be treated as a single emission component. A similar conclusion is reached by Abdo et al. (2009d) for an HMXB (LS I +61 303), based on the light curves and spectral incompatibility of this source in the two bands. 


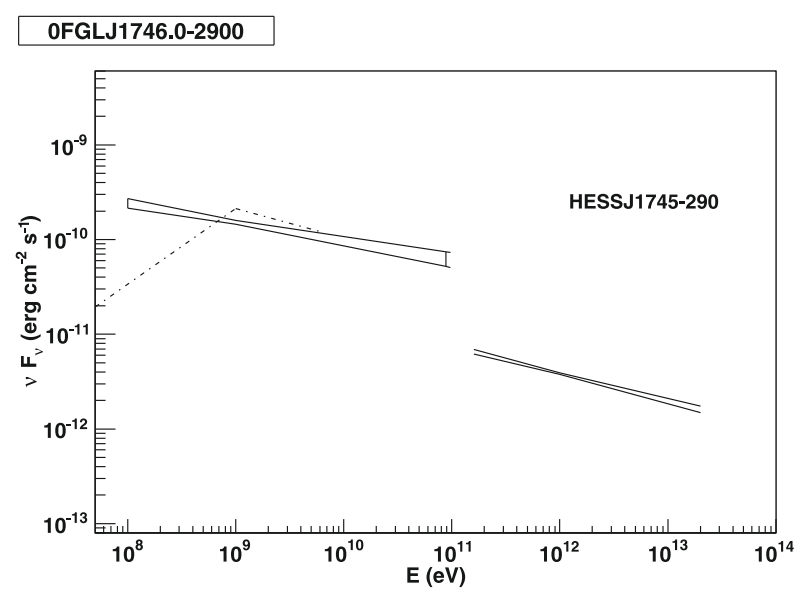

Fig. 9. $\mathrm{MeV}$ to $\mathrm{TeV}$ spectrum of the Galactic center. The VHE spectrum is taken from Aharonian et al. (2006h) while the broken powerlaw spectrum (dashed-dotted line) derived for 3EG J1746-2851 is taken from Bertsch et al. (2000).

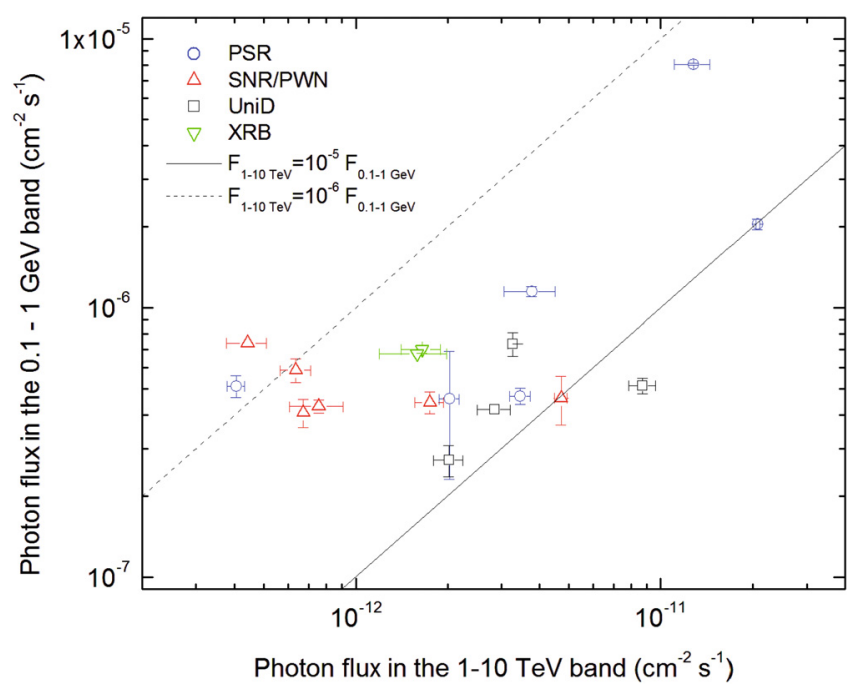

Fig. 10. Photon flux in the $100 \mathrm{MeV}-1 \mathrm{GeV}$ band versus flux in the $1-10 \mathrm{TeV}$ band for coincident $\mathrm{GeV} / \mathrm{TeV}$ sources.

Table 4. HESS sources which have a coincident OFGL source but do not have a 3EG counterpart as in Reimer et al. (2008).

\begin{tabular}{lcc}
\hline \hline HESS sources & 0FGL sources & Coincidence level \\
\hline HESS J1616-508 & 0FGL J1615.6-5049 & $Y$ \\
HESS J1634-472 & 0FGL J1634.9-4737 & $P$ \\
HESS J1745-290 & 0FGL J1746.0-2900 & $P$ \\
HESS J1804-216 & 0FGL J1805.3-2138 & $Y$ \\
HESS J1834-087 & 0FGL J1834.4-0841 & $Y$ \\
HESS J1837-069 & 0FGL J1836.1-0727 & $P$ \\
\hline
\end{tabular}

6. Abdo et al. (2009g) consider a probability that many unidentified LAT sources are extragalactic, so as to explain the low rate of finding coincident MILAGRO emission among the unidentified LAT sources. This idea might also explain the nondetection of VHE counterparts of a majority of the unidentified LAT sources. On the other hand, the extended nature of all the five spatially coincident cases (HESS J1023-575, HESS J1804-216, HESS J1841-055,

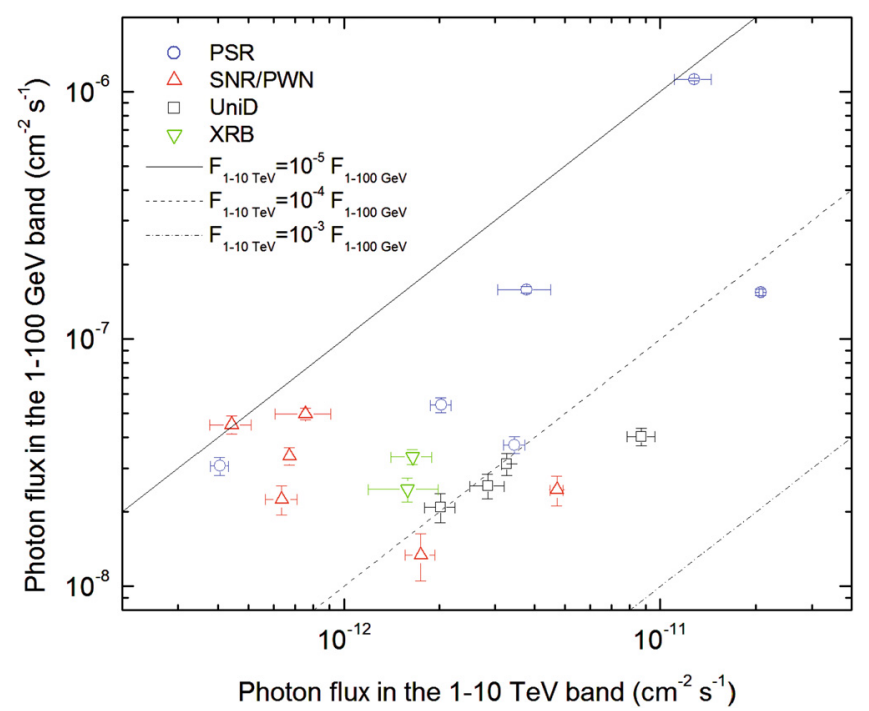

Fig. 11. Photon flux in the $1-100 \mathrm{GeV}$ band versus flux in the $1-10 \mathrm{TeV}$ band for coincident $\mathrm{GeV} / \mathrm{TeV}$ sources.

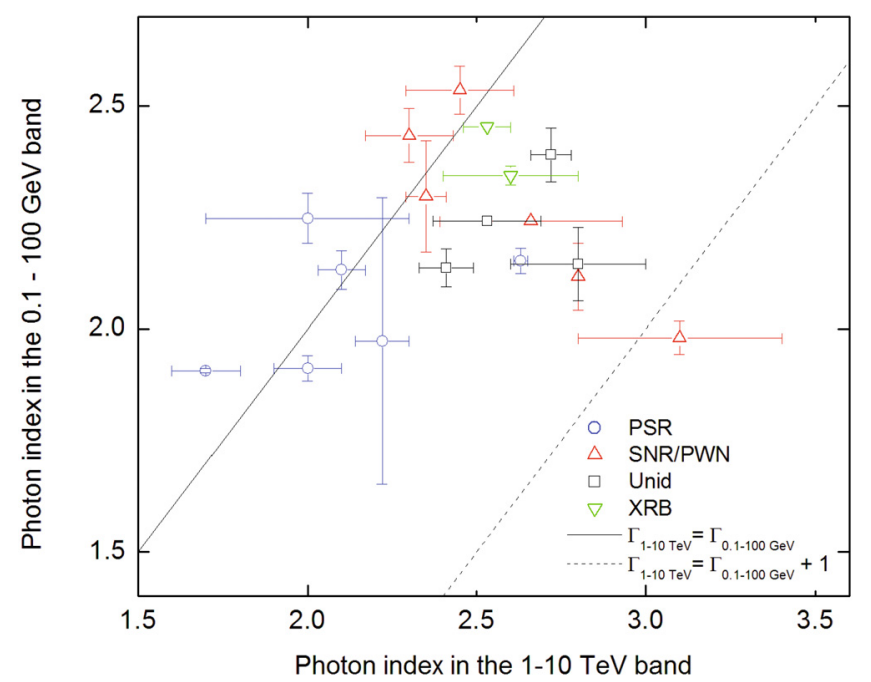

Fig. 12. Photon index in the $0.1-100 \mathrm{GeV}$ band (derived according to Sect. 3.1) versus photon index in the $1-10 \mathrm{TeV}$ band, for coincident $\mathrm{GeV} / \mathrm{TeV}$ sources. OFGL J1923.0+1411/HESS J1923+141 is not included since its VHE photon index is not known.

HESS J1843-033, HESS J1848-018; if proved to be real association) would exclude an extragalactic origin of the corresponding LAT sources.

7. Although VHE observations only cover a small part of the whole sky, they do cover the majority of the inner Galaxy, e.g., the HESS telescopes have surveyed the region of $l=$ $-85^{\circ}$ to $60^{\circ}, b=-3^{\circ}$ to $3^{\circ}$ (Chaves et al. 2009a). In this region, there are 41 Fermi bright sources. Among them, 16 are found to be coincident with a VHE counterpart. This fraction $(\sim 2 / 5)$ is higher than for EGRET where about $1 / 4$ of the EGRET sources (in a smaller region) are found to have a coincident VHE counterpart (Funk et al. 2008). Moreover, the number rises to 21 (out of 41) if possibly coincident cases are included and the fraction becomes $50 \%$. The LAT radii of the $95 \%$ confidence region are in general much smaller than the EGRET error boxes, which further strengthens the case of a higher fraction for LAT. Even though the VHE extension is taken into account in this study (but not in 
Table 5. Number of coincidence cases for each source population (excluding extragalactic sources) in the region $l=-85^{\circ}$ to $60^{\circ}, b=-3^{\circ}$ to $3^{\circ}$.

\begin{tabular}{lcc}
\hline \hline LAT Source class & OFGL sources & $\begin{array}{c}\text { Spatially } \\
\text { coincident cases }^{a}\end{array}$ \\
\hline pulsars & 10 & 4 \\
SNR/PWN candidates & 11 & $6(7)$ \\
Unidentified sources & 19 & $5(9)$ \\
\hline Total $^{b}$ & 41 & $16(21)$ \\
\hline
\end{tabular}

Notes. (a) The numbers in brackets include possibly coincident cases $(P) .{ }^{(b)}$ including LS 5039 .

Funk et al. 2008), the typical extension is of the same order as the LAT positional uncertainties. A breakdown of the number of coincidence cases for each source population in the above-defined region of the HESS Galactic Plane Survey is shown in Table 5.

\section{Conclusion}

In this work, we searched for VHE counterparts of each Galactic $\mathrm{GeV}$ source in the OFGL catalog (Abdo et al. 2009a), based on spatial coincidence. This study benefits significantly from the increased LAT angular resolution and its better sensitivity over previous instruments.

Compared to the EGRET era, not only are there more coincident sources (improvement in quantity), but improvements in quality also start to emerge. With the much better sensitivity of LAT, weaker sources are detected that were unknown in the EGRET era. New relations between the $\mathrm{GeV}$ and $\mathrm{TeV}$ spectra are revealed. A single spectral component is unable to describe some sources detected at both $\mathrm{GeV}$ and TeV energies. Two spectral components may be needed in these cases to accommodate the SEDs, where the VHE flux is higher than a power-law extrapolation from $\mathrm{GeV}$ energies.

A high fraction of Fermi bright sources are found to be spatially coincident with a VHE $\gamma$-ray source. This shows that a common $\mathrm{GeV} / \mathrm{TeV}$ source population exists, a conclusion that is in stark disagreement with Funk et al. (2008) in which EGRET data are used.

Acknowledgements. We thank Emma Oña-Wilhelmi and Werner Hofmann for useful discussions.

\section{References}

Abdo, A. A., Allen, B., Berley, D., et al. (MILAGRO Collaboration) 2007, ApJ, 664, L91

Abdo, A. A., Ackermann, M., Ajello, M., et al. (Fermi/LAT Collaboration) 2009a, ApJS, 183, 46

Abdo, A. A., Ackermann, M., Ajello, M., et al. (Fermi/LAT Collaboration) 2009b, Science, 325, 840

Abdo, A. A., Ackermann, M., Atwood, W. B., et al. (Fermi/LAT Collaboration) 2009c, ApJ, 696, 1084

Abdo, A. A., Ackermann, M., Ajello, M., et al. (Fermi/LAT Collaboration) 2009d, ApJ, 701, L123

Abdo, A. A., Ackermann, M., Ajello, M., et al. (Fermi/LAT Collaboration) 2009e, ApJ, 706, L1

Abdo, A. A., Ackermann, M., Ajello, M., et al. (Fermi/LAT Collaboration) 2009f, ApJ, 706, L56

Abdo, A. A., Allen, B. T., Aune, T., et al. (MILAGRO Collaboration) 2009g, ApJ, 700, L127

Abdo, A. A., Ackermann, M., Ajello, M., et al. (Fermi/LAT Collaboration) 2010, ApJS, 187, 460
Acciari, V. A., Aliu, E., Arlen, T., et al. (VERITAS Collaboration) 2009a, ApJ, 700,1034

Acciari, V. A., Aliu, E., Arlen, T., et al. (VERITAS Collaboration) 2009b, ApJ, 698, L133

Acciari, V. A., Aliu, E., Arlen, T., et al. (VERITAS Collaboration) 2009c, ApJ, 703, L6

Acero, F., Aharonian, F. A., Akhperjanian, A. G., et al. (HESS Collaboration) 2010, MNRAS, 402, 1877

Aharonian, F. A., Akhperjanian, A. G., Aye, K.-M., et al. (HESS Collaboration) 2005a, A\&A, 436, L17

Aharonian, F. A., Akhperjanian, A. G., Beilicke, M., et al. (HEGRA Collaboration) 2005b, A\&A, 431, 197

Aharonian, F. A., Akhperjanian, A. G., Bazer-Bachi, A. R., et al. (HESS Collaboration) 2006a, A\&A, 448, L19

Aharonian, F. A., Akhperjanian, A. G., Bazer-Bachi, A. R., et al. (HESS Collaboration) 2006b, A\&A, 448, L43

Aharonian, F. A., Akhperjanian, A. G., Bazer-Bachi, A. R., et al. (HESS Collaboration) 2006c, Nature, 440, 1018

Aharonian, F. A., Akhperjanian, A. G., Bazer-Bachi, A. R., et al. (HESS Collaboration) 2006d, A\&A, 457, 899

Aharonian, F. A., Akhperjanian, A. G., Bazer-Bachi, A. R., et al. (HESS Collaboration) 2006e, A\&A, 460, 743

Aharonian, F. A., Akhperjanian, A. G., Bazer-Bachi, A. R., et al. (HESS Collaboration) 2006f, ApJ, 636, 777

Aharonian, F. A., Akhperjanian, A. G., Bazer-Bachi, A. R., et al. (HESS Collaboration) 2006g, A\&A, 456, 245

Aharonian, F. A., Akhperjanian, A. G., Bazer-Bachi, A. R., et al. (HESS Collaboration) 2006h, Phys. Rev. Lett., 97, 221102

Aharonian, F. A., Akhperjanian, A. G., Bazer-Bachi, A. R., et al. (HESS Collaboration) 2007a, A\&A, 467, 1075

Aharonian, F. A., Akhperjanian, A. G., Bazer-Bachi, A. R., et al. (HESS Collaboration) 2007b, A\&A, 472, 489

Aharonian, F. A., Akhperjanian, A. G., Barres de Almeida, U., et al. (HESS Collaboration) 2008a, A\&A, 477, 481

Aharonian, F. A., Akhperjanian, A. G., Barres de Almeida, U., et al. (HESS Collaboration) 2008b, A\&A, 490, 685

Aharonian, F. A., Akhperjanian, A. G., Barres de Almeida, U., et al. (HESS Collaboration) 2008c, A\&A, 481, 401

Aharonian, F. A., Akhperjanian, A. G., Barres de Almeida, U., et al. (HESS Collaboration) 2008d, A\&A, 477, 353

Aharonian, F., Buckley, J., Kifune, T., \& Sinnis, G. 2008e, Rep. Prog. Phys., 71, 096901

Aharonian, F. A., Akhperjanian, A. G., Anton, G., et al. (HESS Collaboration) 2009, A\&A, 499, 723

Albert, J., Aliu, E., Anderhub, H., et al. (MAGIC Collaboration) 2007, ApJ, 664, L87

Albert, J., Aliu, E., Anderhub, H., et al. (MAGIC Collaboration) 2008, ApJ, 674, 1037

Albert, J., Aliu, E., Anderhub, H., et al. (MAGIC Collaboration) 2009, ApJ, 693, 303

Aliu, E., Anderhub, H., Antonelli, L. A., et al. (MAGIC Collaboration) 2008, Science, 322, 1221

Aliu, E., for the VERITAS Collaboration 2009, AIP Conf. Proc., 1085, 324

Atwood, W. B., Abdo, A. A., Ackermann, M., et al. (Fermi/LAT Collaboration) 2009, ApJ, 697, 1071

Berge, D., Funk, S., \& Hinton, J. A. 2007, A\&A, 466, 1219

Bertsch, D. L., Brazier, K. T. S., Fichtel, C. E., et al. 1992, Nature, 357, 306

Bertsch, D. L., Hartman, R. C., Hunter, S. D., et al. 2000, AIP Conf. Ser., 510, 504

Celik, O., on behalf of the Fermi-LAT Collaboration 2009, Proc. 31st International Cosmic Ray Conference, in press

Chaves, R. C. G., on behalf of the HESS Collaboration 2009a, Proc. 31st International Cosmic Ray Conference, in press

Chaves, R. C. G., de Oña Wilhemi, E., \& Hoppe, S., for the HESS Collaboration 2009b, AIP Conf. Proc., 1085, 372

Djannati-Atai, A., de Jager, O. C., Terrier, R., Gallant, Y. A., Hoppe, S., for the HESS Collaboration 2008a, Proc. 30th International Cosmic Ray Conference; Universidad Nacional Autónoma de México, Mexico City, Mexico, 2, 823

Djannati-Atai, A., Oña-Wilhelmi, E., Renaud, M., Hoppe, S., for the HESS Collaboration 2008b, Proc. 30th International Cosmic Ray Conference; Universidad Nacional Autónoma de México, Mexico City, Mexico, 2, 863

Dubois, F., Glück, B., de Jager, O. C., et al. 2009, Proc. 31st ICRC, Łódź, Poland Feldman, G. J., \& Cousins, R. D. 1998, Phys. Rev. D., 57, 3873

Fiasson, A., Marandon, V., Chaves, R. C. G., Tibolla, O., for the HESS Collaboration 2009, Proc. 31st International Cosmic Ray Conference, in press

Finnegan, G., on behalf of the VERITAS Collaboration 2009, Proc. 31st International Cosmic Ray Conference, in press

Funk, S., Hermann, G., Hinton, J. A. et al. 2004, Astropart. Phys., 22, 285 
Funk, S., Reimer, O., Torres, D. F., \& Hinton, J. A. 2008, ApJ, 679, 1299

Gargano, F. for the Fermi/LAT Collaboration 2009, Proc. 31st International Cosmic Ray Conference, in press

Grondin, M.-H., on behalf of the Fermi-LAT Collaboration 2009, Proceedings of the 31st International Cosmic Ray Conference, in press

Halpern, J. P., Camilo, F., Gotthelf, E. V., et al. 2001, ApJ, 552, L125

Hartman, R. C., Bertsch, D. L., Bloom, S. D., et al. 1999, ApJS, 123, 79

Hillas, A. M. 1996, Space Sci. Rev., 75, 17

Hoppe, S., for the HESS Collaboration, 2008, Proc. 30th International Cosmic Ray Conference; Universidad Nacional Autónoma de México, Mexico City, Mexico, 2, 579, [arXiv:0710.3528]

Hoppe, S., de Oña Wilhemi, E., Khélifi, B., et al. for the HESS Collaboration 2009, Proc. 31st International Cosmic Ray Conference, in press [arXiv:0906.5574]

Hinton, J. 2009, New J. Phys., 11, 055005

de Jager, O. C., Harding, A. K., Michelson, P. F., et al. 1996, ApJ, 457, 253

Lamb, R. C., \& Macomb, D. J. 1997, ApJ, 488, 872

Lemoine-Goumard, M., \& Grondin, M.-H., on behalf of the Fermi-LAT Collaboration and the Pulsar Timing Consortium, 2009, Proc. 31st International Cosmic Ray Conference, in press

Li, T.-P., \& Ma, Y.-Q. 1983, ApJ, 272, 317

Mayer-Hasselwander, H. A., Bertsch, D. L., Brazier, K. T. S., et al. 1994, ApJ, 421,276
Ng, C.-Y., Roberts, M. S. E., \& Romani, R. W. 2005, ApJ, 627, 904

Ohm, S., Horns, D., Reimer, O., et al., for the HESS Collaboration 2009, HEPIMS Workshop (Jaen 2009), Publications of the AS, in press [arXiv:0906.2637]

Ong, R. A., Acciari, V. A., Arlen, T., for the VERITAS collaboration 2009, Proc. 2009 Fermi Symposium, in press [arXiv: 0912 . 5355]

Reimer, O., Funk, S., Torres, D. F., \& Hinton, J. 2008, Proc. 30th International Cosmic Ray Conference; Universidad Nacional Autónoma de México, Mexico City, Mexico, 2, 613

de los Reyes, R., Bednarek, W., Camara, M., \& Lopez, M., for the MAGIC Collaboration 2009, Proc. 31st International Cosmic Ray Conference, in press Thompson, D. J., Bailes, M., Bertsch, D. L., et al. 1996, ApJ, 465, 385

Tibolla, O., on behalf of the HESS Collaboration 2009a, AIP Conf. Proceed., 1112, 211

Tibolla, O., Komin, N., Kosack, K., \& Naumann-Godo, M., on behalf of the HESS Collaboration 2009b, AIP Conf. Proceed., 1085, 249

Weinstein, A., for the VERITAS collaboration 2009, Proc. 2009 Fermi Symposium, in press [arXiv: 0912.5355]

Zanin, R., for the MAGIC collaboration 2009, Proc. 2009 Fermi Symposium, in press [arXiv: 0912 . 3671]

Zepka, A., Cordes, J. M., Wasserman, I., \& Lundgren, S. C. 1996, ApJ, 456, 305 